\title{
On a Generalisation of the Laplace Transformation
}

\author{
By A. ERdélyi
}

(Received 13th June 1950. Read 3rd November 1950.)

1. On reading a recent paper by R. S. Varma (Varma 1949) I recalled that in May $1942 \mathrm{I}$ investigated an integral transformation which is very similar to Varma's. Varma has

$$
\phi_{m}^{k}(s t)=\int_{0}^{\infty}(2 s t)^{-t} W_{k, m}(2 s t) f(t) d t
$$

and points out that this reduces to a Laplace integral for $k=\frac{1}{4}$, $m= \pm \frac{1}{4}$. Instead of (1), one could consider the integral

$$
\int_{0}^{\infty} e^{-\frac{k}{s} t} W_{k, m}(s i)(s t)^{-k} f(t) d t
$$

which was introduced by C. S. Meijer (Meijer $1940 \mathrm{~b}$ ); this integral reduces to a Laplace integral whenever $k=m+\frac{1}{2}$. Now, apart from comparatively unimportant factors, the nucleus of (2) is a fractional derivative or integral, as the case may be, of $e^{-s t}$, and on carrying out a fractional integration by parts, it appears that (2) is essentially the Laplace transform of a fractional integral.or derivative of $f$. Thus, the whole theory of the transformation (2), including inversion formulae, representation theorems, etc., can be deduced from the well-known theory of the Laplace transformation. It is not quite clear that a similar reduction is possible for (1), although it is certainly possible when $k=0$.

My work of 1942 remained unpublished, and I still hope to describe it in more detail on some future occasion. Meanwhile, in view of the reviving interest in the subject, ${ }^{1}$ I should like to establish briefly the connection between (2) and the Laplace transformation.

2. I shall use the operators of fractional integration and differentiation whose theory has been developed by H. Kober and myself. For the sake of brevity, I shall formulate all results for the class $L_{2}(0, \infty)$ and merely remark that corresponding results are known for the classes $L_{p}, 1 \leqq p \leqq \infty$. The definition of the operators in the simplest case is (Kober 1940)

1 Cf. for instance, a series of papers by S. K. Bose, a pupil of Dr Varma's. 


$$
\begin{aligned}
& I_{\eta, a}^{+} f(x)=\{\Gamma(a)\}^{-1} x^{-\eta-a} \int_{0}^{x}(x-u)^{a-1} u^{\eta} f(u) d u \\
& K_{\eta, a}^{-} f(x)=\{\Gamma(a)\}^{-1} x^{\eta} \int_{x}^{\infty}(u-x)^{a-1} u^{-\eta-a} f(u) d u
\end{aligned}
$$

where $f(x)$ is in $L_{2}(0, \infty)$, Re $a>0, \operatorname{Re} \eta>-\frac{1}{2}$.

First, the definitions can be extended to other values of $\eta$, as long as Re $\eta-\frac{1}{2}$ is not a negative integer (Erdélyi 1940); next follows the extension to Re $a=0$ which is much more difficult (Kober 1941). The domain of these extensions is still the full class $L_{2}$. Lastly, the extension to $\operatorname{Re} a<0$ is given by the definition

$$
I_{\eta, a}^{+}=\left(I_{\eta+\alpha,-a}^{+}\right)^{-1}, \quad K_{\eta, a}=\left(K_{\eta+a,-a}\right)^{-1} \text {. }
$$

In this last extension it is necessary to contract the domain of definition from the full $L_{2}$ to a class $L_{2}^{(a)}$ which coincides with $L^{2}$ if Re $a \geqq 0$. Here the operators will be used in the extended sense (Erdélyi 1940).

We define the Mellin transform as

$$
\mathfrak{f t}_{t} f(x)=\underset{X \rightarrow \infty}{1 . \text { i. m. }} \int_{X^{-1}}^{X} x^{-1+i t} f(x) d x,
$$

where the right-hand side is a limit in mean square. For the extended operators we then have

$$
\begin{aligned}
& \mathfrak{A l}_{t} I_{\eta, a}^{+} f=\frac{\Gamma\left(\eta+\frac{1}{2}-i t\right)}{\Gamma\left(\eta+a+\frac{1}{2}-i t\right)} \mathfrak{A t}_{t} f \\
& \mathfrak{H I}_{\iota} K_{\eta, a}^{-} f=\frac{\Gamma\left(\eta+\frac{1}{2}+i t\right)}{\Gamma\left(\eta+a+\frac{1}{2}+i t\right)} \mathfrak{A t}_{t} f .
\end{aligned}
$$

Moreover, we have the formula for fractional integration by parts

$$
\int_{0}^{\infty} d x \phi(x) I_{\eta, a}^{+} f(x)=\int_{0}^{\infty} d x f(x) K_{\eta, \alpha}^{-} \phi(x),
$$

valid if both $f(x)$ and $\phi(x)$ belong to $L_{2}^{(\alpha)}$.

3. After these preliminaries we define the nucleus of our transform

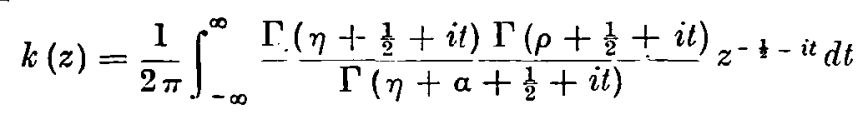

where we assume that neither $\operatorname{Re} \eta-\frac{1}{2}$ nor $\operatorname{Re} \rho-\frac{1}{2}$ is a negative integer. The evaluation of (8) as the sum of residues leads to an expression in terms of confluent hypergeometric functions or "cut" confluent hypergeometric functions; and from Mellin's inversion formula we have

$$
\mathfrak{H}_{t} k(z)=\frac{\Gamma\left(\eta+\frac{1}{2}+i t\right) \Gamma\left(\rho+\frac{1}{2}+i t\right)}{\Gamma\left(\eta+a+\frac{1}{2}+i t\right)}=\frac{\Gamma\left(\eta+\frac{1}{2}+i t\right)}{\Gamma\left(\eta+\alpha+\frac{1}{2}+i t\right)} \mathbb{H}_{t}\left(z^{\rho} e^{-z}\right),
$$


and thus

$$
k(z)=K_{\eta, a}^{-}\left(z^{\rho} e^{-z}\right) .
$$

We can now integrate by parts according to $(7)$ and find on account of (9) that

$$
g(x)=\int_{0}^{\infty} k(x y) f(y) d y=\int_{0}^{\infty} e^{-x y}(x y)^{\rho} I_{\eta, a}^{+} f(y) d y
$$

and hence the reduction of the $k$-transform of $f$ (in $L_{2}^{(a)}$ ) to $x^{\rho}$ times. the Laplace transform of $y^{\rho} I_{\eta, a}^{+} f(y)$. It is also possible to prove (although this requires a justification, by absolute convergence, of the interchange of the order of integrations) that for the function defined by $(10)$

$$
K_{\eta+a_{,-a}}^{-} g(x)=\int_{0}^{\infty} e^{-x y}(x y)^{\rho} f(y) d y
$$

for all functions $f$ in $L_{2}$. This latter form enables one to invert the $k$-transformation by means of any of the numerous inversion formulae of the Laplace transformation. For representation theorems, (10) is the more suitable form.

In my unpublished work, I developed the theory of the more general transformation whose nucleus is

$$
\frac{1}{2 \pi} \int_{-\infty}^{\infty} \frac{\Gamma\left\{\left(\eta+\frac{1}{2}+i t\right) / n\right\} \Gamma\left(\rho+\frac{1}{2}+i t\right)}{\Gamma\left\{a+\left(\eta+\frac{1}{2}+i t\right) / n\right\}}(x y)^{-1-i t} d t
$$

where $n$ is any positive number, not necessarily integer. $n=1$ is the nucleus (8), $n=2$ leads to the particular case $k=0$ of (1): this particular case has been studied in some detail (Meijer 1940a, Boas $19+2 a, b)$.

[Added 20th September 1454. Since this note was submitted for publication, a further instalment has appeared in Rend. Sem. Mat. Università e Politecnico di Torino 10 (1950/51), 217-234. A transformation which is equivalent to (2) has also been investigated by K. P. Bhatnagar, Ganita 3 (195\%), 13-18, who refers to unpublished work by R. S. Varma.]

\section{REFERENCES.}

Boas, R. P., 1942a. Proc. Nut. Ac. Sci. 28, 21-24.

1942b. Bull. American Math. Sor. 48, 286-294.

Erdélyi, A., 1940. Quart. J. Math. (Oxford) 11, 293-303

Kober, H., 1940. Quart. J. Math. (Oxford) 11, 193-211.

1941. Trans. American Math. Soc. 50, 160-174.

Meijer, C. S., 1940a. Proc. Amsterdam Ak. Wet. 43, 599-608 and 702-711.

1940b. Proc. A msterdam Ak. Wet. 44, 727-737 and 831-839.

Varma, R. S., 1949. Proc. Edinburgh Math. Soc. (2) 8, 126-127.

California Institute of Technology, Pasadena 4, California. 